\title{
Hydration Dependent Structural Deformation of Guanine in the Electronic Singlet Excited State
}

$\underline{\text { Supporting Information }}$

\author{
M.K. Shukla and Jerzy Leszczynski*
}

Table S1. Bond lengths ( $\AA$ ) of guanine in the isolated and hydrated complexes in the ground and lowest singlet $\pi \pi^{*}$ excited state obtained at the HF/6-311G(d,p) and CIS/6$\underline{311 \mathrm{G}(\mathrm{d}, \mathrm{p}) \text { levels, respectively. }}$.

\begin{tabular}{|c|c|c|c|c|c|c|c|c|c|c|}
\hline \multicolumn{3}{|c|}{$\mathrm{G}^{*}$} & & & \multicolumn{2}{|l|}{$\mathrm{G}+1 \mathrm{~W}$} & \multicolumn{2}{|l|}{$\mathrm{G}+3 \mathrm{~W}$} & \multicolumn{2}{|l|}{$\mathrm{G}+5 \mathrm{~W}$} \\
\hline Bond & $\mathrm{S}_{0}$ & $\pi \pi^{*}$ & & & $\mathrm{~S}_{0}$ & $\pi \pi^{*}$ & $\mathrm{~S}_{0}$ & $\pi \pi^{*}$ & $\mathrm{~S}_{0}$ & $\pi \pi^{*}$ \\
\hline & $\mathrm{HF}$ & CIS & $\mathrm{TD}^{\mathrm{a}}$ & $\mathrm{CAS}^{\mathrm{b}}$ & $\mathrm{HF}$ & CIS & $\mathrm{HF}$ & CIS & $\mathrm{HF}$ & CIS \\
\hline $\mathrm{N} 1 \mathrm{C} 2$ & 1.357 & 1.397 & 1.399 & 1.387 & 1.356 & 1.397 & 1.354 & .399 & 1.360 & 1.375 \\
\hline C2N3 & 1.286 & & 1.475 & 1.465 & 1.290 & & 93 & & 306 & \\
\hline N3C4 & 1.355 & 1.284 & 1.279 & 1.279 & 1.351 & & 57 & 287 & 1.348 & 32 \\
\hline C4C5 & 1.367 & 1.431 & 1.476 & 1.466 & 1.369 & & 1.369 & & 1.369 & 1.468 \\
\hline C5C6 & 1.436 & 1.455 & 1.481 & 1.473 & 1.430 & & 1.428 & & 1.426 & 1.417 \\
\hline C6N1 & 1.417 & 1.429 & 1.416 & 1.399 & 1.407 & & 1.403 & & 1.395 & 1.422 \\
\hline C5N7 & 1.378 & 1.354 & 1.359 & 1.381 & 1.380 & 1. & 1.379 & 1.352 & 1.382 & 1.351 \\
\hline N7C8 & 1.276 & 1.293 & 1.312 & 1.294 & 1.275 & & 1.278 & & 1.275 & 1.296 \\
\hline C8N9 & 75 & & & & 1.376 & & & & 76 & 53 \\
\hline N9C4 & 52 & & 6 & & 1.352 & & & & 53 & 11 \\
\hline C6O & 88 & & 0 & & 1.198 & & & & .205 & 13 \\
\hline $\mathrm{C} 2 \mathrm{~N} 2$ & 1.362 & 1.370 & 1.362 & 1.371 & 1.356 & & & & 1.330 & 1.320 \\
\hline $\mathrm{N} 1 \mathrm{H}$ & 0.996 & 0.998 & 1.023 & 0.9 & 1.002 & & 1.003 & & 1.001 & 0.999 \\
\hline $\mathrm{N} 2 \mathrm{H} 21$ & 0.994 & & 10 & 0. & 0.993 & & 0.9 & & 95 & \\
\hline $\mathrm{N} 2 \mathrm{H} 2$ & 5 & & & & 0.993 & & 3 & & 1 & \\
\hline $\mathrm{C} 8 \mathrm{H}$ & 71 & & & & 1.071 & & & & 71 & 73 \\
\hline \multirow[t]{2}{*}{$\mathrm{N} 9 \mathrm{H}$} & 0.992 & & 1.007 & & 0.993 & 0.992 & 0.999 & & 0.994 & 0.994 \\
\hline & \multicolumn{2}{|l|}{ G+6W } & \multicolumn{2}{|c|}{$\mathrm{G}+7 \mathrm{~W} 1$} & \multicolumn{2}{|c|}{$\mathrm{G}+7 \mathrm{~W} 1+\mathrm{PCM}$} & \multicolumn{2}{|l|}{$\mathrm{G}+7 \mathrm{~W} 2$} & \multicolumn{2}{|c|}{$\mathrm{G}+7 \mathrm{~W} 3$} \\
\hline \multirow[t]{2}{*}{ Bond } & $\mathrm{S}_{0}$ & $\pi \pi^{*}$ & $\mathrm{~S}_{0}$ & $\pi \pi^{*}$ & $\overline{S_{0}}$ & $\pi \pi^{*}$ & $\mathrm{~S}_{0}$ & . & $\mathrm{S}_{0}$ & $*$ \\
\hline & $\mathrm{HF}$ & CIS & $\mathrm{HF}$ & CIS & HF & CIS & $\mathrm{HF}$ & CIS & $\mathrm{HF}$ & CIS \\
\hline $\mathrm{N} 1 \mathrm{C} 2$ & 1.359 & 1.37 & 1.359 & & 1.361 & 3 & 1.358 & & 1.359 & 1.383 \\
\hline $\mathrm{C} 2 \mathrm{~N} 3$ & 1.305 & 1.3 & 1.306 & 1.3 & 1.307 & & 1.2 & & 1.304 & 1.333 \\
\hline $\mathrm{N} 3 \mathrm{C} 4$ & 1.354 & 1.3 & & & 1.3 & & 1. & & 56 & \\
\hline $\mathrm{C} 4 \mathrm{C} 5$ & 1.371 & 1.464 & 1.3 & 1.4 & 1.373 & & 71 & & 1.369 & 1. \\
\hline C5C6 & 1.425 & 1.417 & 1.425 & 1.4 & 1.417 & & 24 & 17 & 25 & 1.418 \\
\hline C6N1 & 1.395 & 1.429 & 1.392 & & 1.384 & & 1.396 & & 1.398 & 1.434 \\
\hline C5N7 & 1.380 & 1.349 & 1.381 & 1.351 & 1.385 & & 1.381 & 1.349 & 1.380 & 1.348 \\
\hline N7C8 & 1.277 & 1.304 & 1.278 & & 1.282 & & 1.278 & & 1.281 & 1.310 \\
\hline & 1.371 & 1.341 & 1.370 & & 1.369 & & 1.372 & & 1.369 & 1.341 \\
\hline N9C4 & 1.350 & 1.397 & 1.351 & 1.4 & 1.349 & & 1.349 & & 51 & 1.394 \\
\hline C6O & 1.206 & 1.215 & 1.206 & 1.215 & 1.218 & & 1.206 & 1.216 & 1.203 & 1.212 \\
\hline $\mathrm{C} 2 \mathrm{~N} 2$ & 1.333 & 1.325 & 1.334 & & 1.330 & & 1.334 & 1.326 & 1.334 & 1.328 \\
\hline $\mathrm{N} 1 \mathrm{H}$ & 1.001 & 0.999 & 1.001 & & 1.004 & & 1.001 & 0.999 & 1.001 & 0.999 \\
\hline $\mathrm{N} 2 \mathrm{H} 21$ & 0.995 & 0.998 & 0.996 & 0.9 & 0.996 & & 0.995 & 0.998 & 0.994 & 0.998 \\
\hline $\mathrm{N} 2 \mathrm{H} 2$ & 0.999 & 1.000 & & & 1.000 & & 0.999 & & 0.999 & 1.000 \\
\hline & 1.071 & 1.072 & & & 1.075 & & 1.069 & & 1.072 & 1.073 \\
\hline $\mathrm{N9H}$ & 1.003 & 1.002 & 1.003 & 1.002 & 1.006 & 1.005 & 0.998 & 0.997 & 1.003 & 1.002 \\
\hline
\end{tabular}




\begin{tabular}{|c|c|c|c|c|c|c|c|c|c|c|}
\hline & \multicolumn{2}{|c|}{$\mathrm{G}+8 \mathrm{~W}$} & \multicolumn{2}{|c|}{$\mathrm{G}+8 \mathrm{~W}+\mathrm{PCM}$} & \multicolumn{2}{|l|}{$\mathrm{G}+9 \mathrm{~W}$} & \multicolumn{2}{|l|}{$\mathrm{G}+10 \mathrm{~W}$} & \multicolumn{2}{|c|}{$\mathrm{G}+11 \mathrm{~W} 1$} \\
\hline \multirow[t]{2}{*}{ Bond } & $\mathrm{S}_{0}$ & $\pi \pi^{*}$ & $\mathrm{~S}_{0}$ & $\pi \pi^{*}$ & $\mathrm{~S}_{0}$ & $\pi \pi^{*}$ & $\mathrm{~S}_{0}$ & $\pi \pi^{*}$ & $\mathrm{~S}_{0}$ & $\pi \pi^{*}$ \\
\hline & $\mathrm{HF}$ & CIS & $\mathrm{HF}$ & CIS & $\mathrm{HF}$ & CIS & $\mathrm{HF}$ & CIS & $\mathrm{HF}$ & CIS \\
\hline N1C2 & 1.365 & 1.375 & 1.364 & 1.381 & 1.356 & 1.370 & 1.360 & 1.369 & 1.360 & 1.410 \\
\hline $\mathrm{C} 2 \mathrm{~N} 3$ & 1.309 & 1.330 & 1.307 & 1.325 & 1.306 & 1.331 & 1.306 & 1.331 & 1.307 & 1.409 \\
\hline N3C4 & 1.352 & 1.331 & 1.348 & 1.332 & 1.352 & 1.335 & 1.354 & 1.335 & 1.355 & 1.287 \\
\hline $\mathrm{C} 4 \mathrm{C} 5$ & 1.370 & 1.467 & 1.372 & 1.467 & 1.368 & 1.475 & 1.369 & 1.474 & 1.368 & 1.424 \\
\hline C5C6 & 1.419 & 1.414 & 1.416 & 1.408 & 1.426 & 1.414 & 1.424 & 1.414 & 1.420 & 1.464 \\
\hline C6N1 & 1.394 & 1.423 & 1.387 & 1.417 & 1.394 & 1.421 & 1.393 & 1.421 & 1.394 & 1.383 \\
\hline C5N7 & 1.380 & 1.351 & 1.385 & 1.353 & 1.381 & 1.351 & 1.381 & 1.351 & 1.379 & 1.350 \\
\hline N7C8 & 1.277 & 1.304 & 1.282 & 1.314 & 1.279 & 1.303 & 1.279 & 1.303 & 1.281 & 1.306 \\
\hline C8N9 & 1.374 & 1.341 & 1.369 & 1.332 & 1.371 & 1.340 & 1.370 & 1.341 & 1.369 & 1.347 \\
\hline N9C4 & 1.350 & 1.400 & 1.349 & 1.398 & 1.350 & 1.404 & 1.350 & 1.405 & 1.351 & 1.372 \\
\hline $\mathrm{C} 6 \mathrm{O}$ & 1.207 & 1.216 & 1.217 & 1.229 & 1.206 & 1.214 & 1.206 & 1.215 & 1.204 & 1.197 \\
\hline $\mathrm{C} 2 \mathrm{~N} 2$ & 1.330 & 1.324 & 1.333 & 1.321 & 1.337 & 1.320 & 1.338 & 1.321 & 1.335 & 1.367 \\
\hline $\mathrm{N} 1 \mathrm{H}$ & 1.002 & 1.000 & 1.005 & 1.000 & 1.005 & 1.002 & 1.005 & 1.003 & 1.001 & 1.006 \\
\hline $\mathrm{N} 2 \mathrm{H} 21$ & 1.000 & 0.999 & 1.000 & 0.999 & 1.004 & 1.004 & 1.005 & 1.005 & 1.005 & 1.001 \\
\hline $\mathrm{N} 2 \mathrm{H} 22$ & 0.999 & 1.000 & 1.000 & 1.002 & 0.995 & 0.996 & 0.994 & 0.996 & 0.992 & 0.997 \\
\hline $\mathrm{C} 8 \mathrm{H}$ & 1.071 & 1.072 & 1.075 & 1.079 & 1.071 & 1.073 & 1.071 & 1.073 & 1.071 & 1.072 \\
\hline \multirow[t]{2}{*}{$\mathrm{N} 9 \mathrm{H}$} & 1.003 & 1.002 & 1.006 & 1.005 & 1.002 & 1.001 & 1.004 & 1.004 & 1.005 & 1.007 \\
\hline & \multicolumn{2}{|c|}{$\mathrm{G}+11 \mathrm{~W} 2$} & \multicolumn{2}{|c|}{$\mathrm{G}+12 \mathrm{~W}$} & \multicolumn{2}{|c|}{$\mathrm{G}+13 \mathrm{~W}$} & & & & \\
\hline \multirow[t]{2}{*}{ Bond } & $\mathrm{S}_{0}$ & $\pi \pi^{*}$ & $\mathrm{~S}_{0}$ & $\pi \pi^{*}$ & $\mathrm{~S}_{0}$ & $\pi \pi^{*}$ & & & & \\
\hline & $\mathrm{HF}$ & CIS & $\mathrm{HF}$ & CIS & $\mathrm{HF}$ & CIS & & & & \\
\hline N1C2 & 1.360 & 1.403 & 1.361 & 1.410 & 1.360 & 1.402 & & & & \\
\hline $\mathrm{C} 2 \mathrm{~N} 3$ & 1.293 & 1.355 & 1.307 & 1.408 & 1.296 & 1.350 & & & & \\
\hline N3C4 & 1.353 & 1.294 & 1.354 & 1.287 & 1.352 & 1.298 & & & & \\
\hline C4C5 & 1.372 & 1.433 & 1.368 & 1.425 & 1.372 & 1.435 & & & & \\
\hline C5C6 & 1.416 & 1.434 & 1.418 & 1.464 & 1.417 & 1.431 & & & & \\
\hline C6N1 & 1.394 & 1.430 & 1.394 & 1.383 & 1.395 & 1.433 & & & & \\
\hline C5N7 & 1.381 & 1.347 & 1.380 & 1.350 & 1.382 & 1.346 & & & & \\
\hline N7C8 & 1.282 & 1.312 & 1.283 & 1.308 & 1.281 & 1.313 & & & & \\
\hline C8N9 & 1.371 & 1.345 & 1.370 & 1.347 & 1.372 & 1.346 & & & & \\
\hline N9C4 & 1.347 & 1.376 & 1.350 & 1.371 & 1.348 & 1.377 & & & & \\
\hline $\mathrm{C} 6 \mathrm{O}$ & 1.212 & 1.215 & 1.207 & 1.198 & 1.211 & 1.218 & & & & \\
\hline $\mathrm{C} 2 \mathrm{~N} 2$ & 1.352 & 1.358 & 1.334 & 1.366 & 1.347 & 1.348 & & & & \\
\hline $\mathrm{N} 1 \mathrm{H}$ & 0.996 & 0.998 & 1.002 & 1.006 & 0.996 & 0.998 & & & & \\
\hline $\mathrm{N} 2 \mathrm{H} 21$ & 0.996 & 0.999 & 1.005 & 1.001 & 0.996 & 0.999 & & & & \\
\hline $\mathrm{N} 2 \mathrm{H} 22$ & 0.999 & 1.001 & 0.991 & 0.997 & 1.001 & 1.002 & & & & \\
\hline $\mathrm{C} 8 \mathrm{H}$ & 1.072 & 1.073 & 1.073 & 1.074 & 1.072 & 1.075 & & & & \\
\hline $\mathrm{N} 9 \mathrm{H}$ & 1.002 & 1.003 & 1.005 & 1.007 & 1.000 & 1.001 & & & & \\
\hline
\end{tabular}

*TD represents TDDFT level [ref. 3f], and CAS represents CASSCF level [ref. 3d]. 
Table S2: Bond angles $\left(^{\circ}\right)$ of isolated guanine in the $S_{1}\left(\pi \pi^{*}\right)$ excited state obtained at the CIS, TDDFT and CASSCF levels in the gas phase. ${ }^{\text {a }}$

\begin{tabular}{|l|l|l|l|}
\hline Bond Angles & CIS & TDDFT & CASSCF \\
\hline N1C2N3 & 118.4 & 118.2 & 115.4 \\
\hline C2N3C4 & 109.1 & 100.0 & 101.8 \\
\hline N3C4C5 & 125.1 & 124.4 & 123.2 \\
\hline C5C6C6 & 119.3 & 117.7 & 117.9 \\
\hline C6N1C2 & 109.9 & 110.5 & 109.9 \\
\hline C6C5N7 & 111.2 & 109.9 & 110.1 \\
\hline C4C5N7 & 110.7 & 127.0 & 125.7 \\
\hline C5N7C8 & 105.2 & 110.1 & 109.4 \\
\hline N7C8N9 & 114.4 & 105.5 & 105.3 \\
\hline C8N9C4 & 106.2 & 114.2 & 114.4 \\
\hline N9C4C5 & 104.0 & 106.7 & 106.6 \\
\hline N9C4N3 & 130.9 & 132.0 & 103.9 \\
\hline O6C6N1 & 121.7 & 123.5 & 132.7 \\
\hline O6C6C5 & 128.4 & 126.0 & 123.1 \\
\hline N2C2N1 & 116.7 & 120.1 & 118.5 \\
\hline N2C2N3 & 120.0 & 115.8 & 114.6 \\
\hline H1N1C2 & 113.9 & 125.0 & 121.8 \\
\hline H1N1C6 & 110.1 & 116.6 & 118.7 \\
\hline H8C8N7 & 124.2 & 124.5 & 124.7 \\
\hline H8C8N9 & 121.4 & 121.3 & 120.8 \\
\hline H9N9C8 & 127.1 & 127.0 & 125.9 \\
\hline H9N9C4 & 126.2 & 125.7 & 125.4 \\
\hline
\end{tabular}

${ }^{\mathrm{a}}$ For TDDFT see ref. $3 \mathrm{f}$ and for CASSCF see ref. $3 \mathrm{~d}$. 
Table S3. Some selected dihedral angles $\left(^{\circ}\right)$ and amino group angles $\left(^{\circ}\right)$ of guanine and different hydrated complexes in the ground $\left(\mathrm{S}_{0}\right)$ and lowest singlet $\pi \pi^{*}$ excited state $\left(\mathrm{S}_{1}\right)$ obtained at the HF/6-311G(d,p) and CIS/6-311G(d,p) levels, respectively.

\begin{tabular}{|c|c|c|c|c|c|c|c|c|c|c|c|c|c|c|c|c|c|c|}
\hline \multirow[b]{2}{*}{ Parameters } & \multicolumn{2}{|l|}{$\mathrm{G}$} & \multicolumn{2}{|c|}{$\mathrm{G}+1 \mathrm{~W}$} & \multicolumn{2}{|c|}{$\mathrm{G}+3 \mathrm{~W}$} & \multicolumn{2}{|c|}{$\mathrm{G}+5 \mathrm{~W}$} & \multicolumn{2}{|l|}{$\mathrm{G}+6 \mathrm{~W}$} & \multicolumn{2}{|c|}{$\mathrm{G}+7 \mathrm{~W} 1$} & \multicolumn{2}{|c|}{$\mathrm{G}+7 \mathrm{~W} 1+\mathrm{PCM}$} & \multicolumn{2}{|l|}{$\mathrm{G}+7 \mathrm{~W} 2$} & \multicolumn{2}{|l|}{$\mathrm{G}+7 \mathrm{~W} 3$} \\
\hline & $\mathrm{S}_{0}$ & $\mathrm{~S}_{1}$ & $\mathrm{~S}_{0}$ & $\mathrm{~S}_{1}$ & $\mathrm{~S}_{0}$ & $\mathrm{~S}_{1}$ & $\mathrm{~S}_{0}$ & $\mathrm{~S}_{1}$ & $\mathrm{~S}_{0}$ & $\mathrm{~S}_{1}$ & $\mathrm{~S}_{0}$ & $\mathrm{~S}_{1}$ & $\mathrm{~S}_{0}$ & $\mathrm{~S}_{1}$ & $\mathrm{~S}_{0}$ & $\mathrm{~S}_{1}$ & $\mathrm{~S}_{0}$ & $\mathrm{~S}_{1}$ \\
\hline $\mathrm{H} 21 \mathrm{~N} 2 \mathrm{C} 2$ & 117.9 & 115.3 & 119.2 & 117.1 & 118.8 & 116.7 & 119.7 & 119.2 & 119.6 & 119.2 & 118.8 & 119.2 & 120.3 & 119.5 & 119.6 & 119.1 & 119.7 & 119.1 \\
\hline $\mathrm{H} 22 \mathrm{~N} 2 \mathrm{C} 2$ & 113.8 & 112.7 & 115.2 & 114.3 & 115.7 & 114.4 & 120.1 & 120.4 & 120.4 & 120.4 & 119.7 & 120.1 & 119.9 & 120.3 & 120.4 & 120.2 & 120.5 & 120.5 \\
\hline $\mathrm{H} 21 \mathrm{~N} 2 \mathrm{H} 22$ & 115.0 & 111.4 & 116.7 & 113.8 & 116.3 & 113.3 & 120.0 & 119.8 & 119.9 & 119.9 & 119.3 & 119.9 & 119.3 & 119.9 & 119.9 & 119.9 & 119.8 & 119.8 \\
\hline $360-\Sigma \mathrm{HNH}$ & 13.3 & 20.6 & 8.9 & 14.8 & 9.2 & 15.6 & 0.2 & 0.6 & 0.1 & 0.5 & 2.2 & 0.8 & 0.5 & 0.3 & 0.1 & 0.8 & 0.0 & 0.6 \\
\hline C6N1C2N3 & -0.6 & 64.0 & -0.9 & -64.7 & 0.2 & -64.8 & 1.3 & 38.1 & 1.3 & 43.0 & 3.1 & 37.1 & 1.2 & 41.3 & 1.5 & 43.9 & 1.1 & 46.5 \\
\hline $\mathrm{N} 1 \mathrm{C} 2 \mathrm{~N} 3 \mathrm{C} 4$ & 0.8 & -44.2 & 0.8 & 39.2 & 0.8 & 42.4 & 0.6 & -1.7 & 0.9 & -6.5 & -1.0 & -2.3 & 0.5 & -0.6 & 0.9 & -7.3 & 0.9 & -10.2 \\
\hline $\mathrm{C} 2 \mathrm{~N} 3 \mathrm{C} 4 \mathrm{C} 5$ & -1.0 & 2.4 & -0.6 & 2.0 & -1.2 & 0.0 & -1.6 & -32.2 & -2.1 & -29.0 & -1.6 & -30.6 & -1.5 & -27.8 & -2.2 & -28.6 & -2.1 & -26.5 \\
\hline $\mathrm{N} 3 \mathrm{C} 4 \mathrm{C} 5 \mathrm{C} 6$ & -0.9 & 18.5 & 0.4 & -18.1 & 0.5 & -18.8 & 0.8 & 32.2 & 1.2 & 29.7 & 2.2 & 31.0 & 0.7 & 28.0 & 1.3 & 29.5 & 1.3 & 28.0 \\
\hline $\mathrm{N} 1 \mathrm{C} 6 \mathrm{C} 5 \mathrm{C} 4$ & -0.4 & 0.6 & -0.3 & -7.3 & 0.5 & -3.3 & 1.0 & 2.6 & 1.0 & 5.4 & -0.2 & 2.2 & 0.9 & 6.0 & 1.0 & 5.8 & 0.7 & 7.1 \\
\hline $\mathrm{N} 2 \mathrm{C} 2 \mathrm{~N} 3 \mathrm{C} 4$ & -177.2 & 161.4 & -177.5 & -159.7 & -177.5 & -160.5 & -179.2 & -177.3 & -179.1 & 179.7 & 179.8 & -177.2 & -179.0 & 179.6 & -179.1 & 179.1 & -179.1 & 177.3 \\
\hline $\mathrm{H} 21 \mathrm{~N} 2 \mathrm{C} 2 \mathrm{~N} 1$ & 30.4 & -42.3 & 22.1 & 31.0 & 23.3 & 34.8 & 6.4 & 10.2 & 5.4 & 10.6 & 14.9 & 14.8 & 7.2 & 8.2 & 6.3 & 11.2 & 5.1 & 10.9 \\
\hline \multirow[t]{2}{*}{$\mathrm{H} 22 \mathrm{~N} 2 \mathrm{C} 2 \mathrm{~N} 1$} & 169.4 & -171.8 & 168.4 & 167.9 & 169.0 & 170.4 & -178.6 & -178.7 & -176.5 & -177.4 & 178.3 & -175.8 & 179.0 & -178.0 & -177.7 & -178.5 & -176.1 & -178.2 \\
\hline & \multicolumn{2}{|c|}{$\mathrm{G}+8 \mathrm{~W}$} & \multicolumn{2}{|c|}{$\mathrm{G}+8 \mathrm{~W}+\mathrm{PCM}$} & \multicolumn{2}{|c|}{$\mathrm{G}+9 \mathrm{~W}$} & \multicolumn{2}{|c|}{$\mathrm{G}+10 \mathrm{~W}$} & \multicolumn{2}{|c|}{$\mathrm{G}+11 \mathrm{~W} 1$} & \multicolumn{2}{|c|}{$\mathrm{G}+11 \mathrm{~W} 2$} & \multicolumn{2}{|c|}{$\mathrm{G}+12 \mathrm{~W}$} & \multicolumn{2}{|l|}{$\mathrm{G}+13 \mathrm{~W}$} & & \\
\hline Parameters & $\mathrm{S}_{0}$ & $\mathrm{~S}_{1}$ & $\mathrm{~S}_{0}$ & $\mathrm{~S}_{1}$ & $\mathrm{~S}_{0}$ & $\mathrm{~S}_{1}$ & $\mathrm{~S}_{0}$ & $\mathrm{~S}_{1}$ & $\mathrm{~S}_{0}$ & $\mathrm{~S}_{1}$ & $\mathrm{~S}_{0}$ & $\mathrm{~S}_{1}$ & $\mathrm{~S}_{0}$ & $\mathrm{~S}_{1}$ & $\mathrm{~S}_{0}$ & $\mathrm{~S}_{1}$ & & \\
\hline $\mathrm{H} 21 \mathrm{~N} 2 \mathrm{C} 2$ & 120.7 & 119.1 & 120.9 & 119.7 & 118.8 & 122.8 & 118.7 & 122.4 & 120.2 & 114.5 & 115.6 & 115.7 & 121.6 & 114.7 & 115.8 & 116.9 & & \\
\hline $\mathrm{H} 22 \mathrm{~N} 2 \mathrm{C} 2$ & 118.9 & 120.2 & 117.9 & 120.2 & 116.6 & 118.6 & 116.8 & 118.6 & 117.1 & 114.2 & 114.7 & 114.4 & 117.0 & 114.3 & 115.3 & 116.1 & & \\
\hline $\mathrm{H} 21 \mathrm{~N} 2 \mathrm{H} 22$ & 118.5 & 120.0 & 117.0 & 119.9 & 114.6 & 116.4 & 114.1 & 116.1 & 116.8 & 114.2 & 113.7 & 112.5 & 117.9 & 114.3 & 113.7 & 113.4 & & \\
\hline $360-\Sigma \mathrm{HNH}$ & 1.9 & 0.7 & 4.2 & 0.2 & 10.0 & 2.2 & 10.4 & 2.9 & 5.9 & 17.1 & 16.0 & 17.4 & 3.5 & 16.7 & 15.2 & 13.6 & & \\
\hline C6N1C2N3 & 0.9 & 34.9 & 0.0 & 34.1 & 0.1 & 32.6 & -0.2 & 32.4 & 0.2 & -67.2 & 0.3 & 65.4 & 1.5 & -67.3 & 3.5 & 63.7 & & \\
\hline $\mathrm{N} 1 \mathrm{C} 2 \mathrm{~N} 3 \mathrm{C} 4$ & 0.8 & -0.9 & 0.6 & -1.9 & -0.2 & 0.2 & -0.5 & 0.6 & 0.9 & 51.4 & -1.9 & -35.4 & -0.6 & 51.2 & -2.3 & -31.5 & & \\
\hline $\mathrm{C} 2 \mathrm{~N} 3 \mathrm{C} 4 \mathrm{C} 5$ & -2.3 & -29.4 & -1.2 & -27.4 & -0.9 & -31.4 & -0.2 & -31.8 & -1.4 & -6.9 & 1.1 & -6.5 & -0.4 & -6.8 & 1.4 & -9.7 & & \\
\hline $\mathrm{N} 3 \mathrm{C} 4 \mathrm{C} 5 \mathrm{C} 6$ & 1.9 & 27.6 & 0.9 & 26.4 & 2.0 & 32.5 & 1.6 & 32.7 & 0.7 & -20.9 & -1.1 & 15.3 & 0.5 & -20.7 & -1.3 & 16.6 & & \\
\hline $\mathrm{N} 1 \mathrm{C} 6 \mathrm{C} 5 \mathrm{C} 4$ & 0.1 & 5.0 & -0.1 & 4.4 & -1.9 & -1.2 & -2.1 & -1.1 & 0.4 & 5.9 & 1.6 & 15.5 & 0.3 & 5.4 & 1.9 & 15.9 & & \\
\hline $\mathrm{N} 2 \mathrm{C} 2 \mathrm{~N} 3 \mathrm{C} 4$ & -178.4 & -175.9 & -177.9 & -177.4 & -178.5 & -174.9 & -178.6 & -174.6 & 179.6 & -164.0 & 179.4 & 161.8 & 178.2 & -164.1 & 178.9 & 162.9 & & \\
\hline $\mathrm{H} 21 \mathrm{~N} 2 \mathrm{C} 2 \mathrm{~N} 1$ & 16.3 & -1.7 & 21.5 & -1.3 & 32.4 & 21.8 & 31.8 & 23.1 & -10.7 & -32.2 & 27.0 & 41.9 & -11.6 & -31.6 & 22.8 & 33.2 & & \\
\hline $\mathrm{H} 22 \mathrm{~N} 2 \mathrm{C} 2 \mathrm{~N} 1$ & -179.7 & -172.6 & 178.1 & -174.8 & 176.0 & -175.8 & 174.8 & -177.0 & -162.6 & -166.6 & 162.4 & 175.1 & -169.9 & -166.4 & 159.0 & 171.4 & & \\
\hline
\end{tabular}



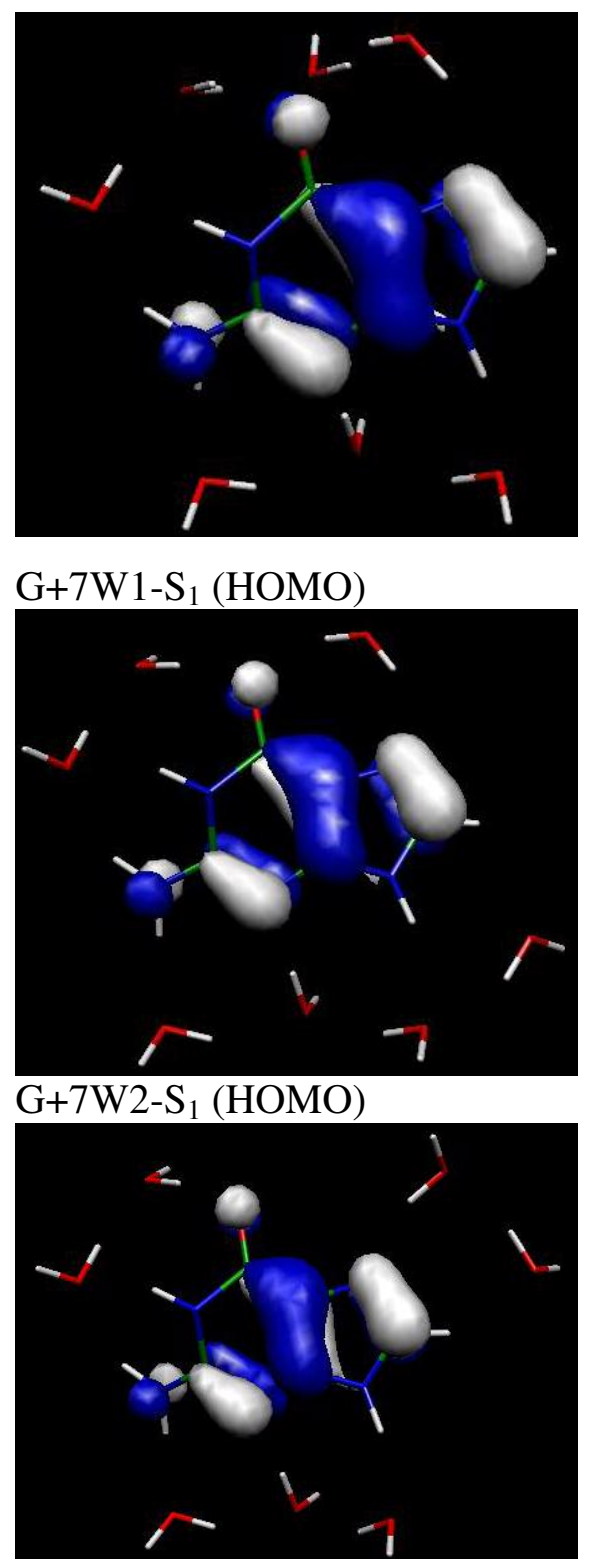

$\mathrm{G}+7 \mathrm{~W} 3-\mathrm{S}_{1}$ (HOMO)

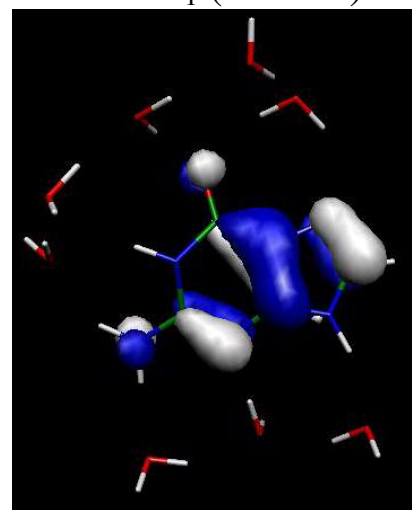

$\mathrm{G}+8 \mathrm{~W}-\mathrm{S}_{1}(\mathrm{HOMO})$
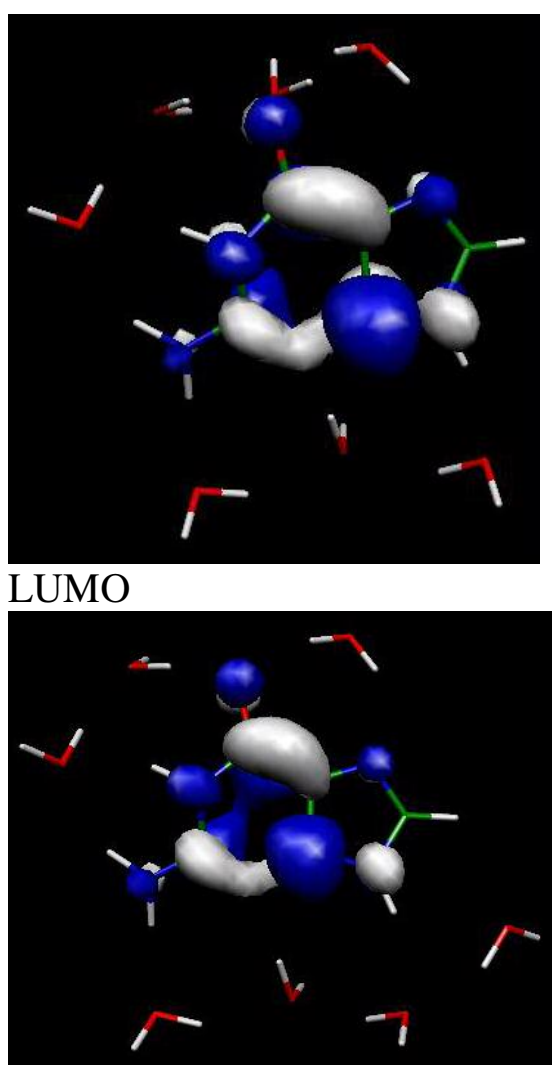

LUMO
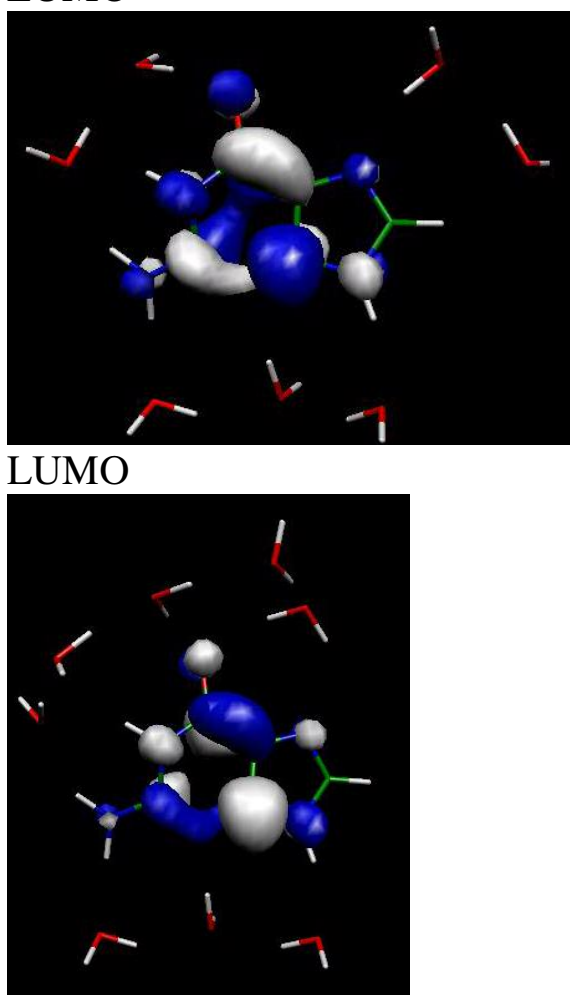

LUMO 

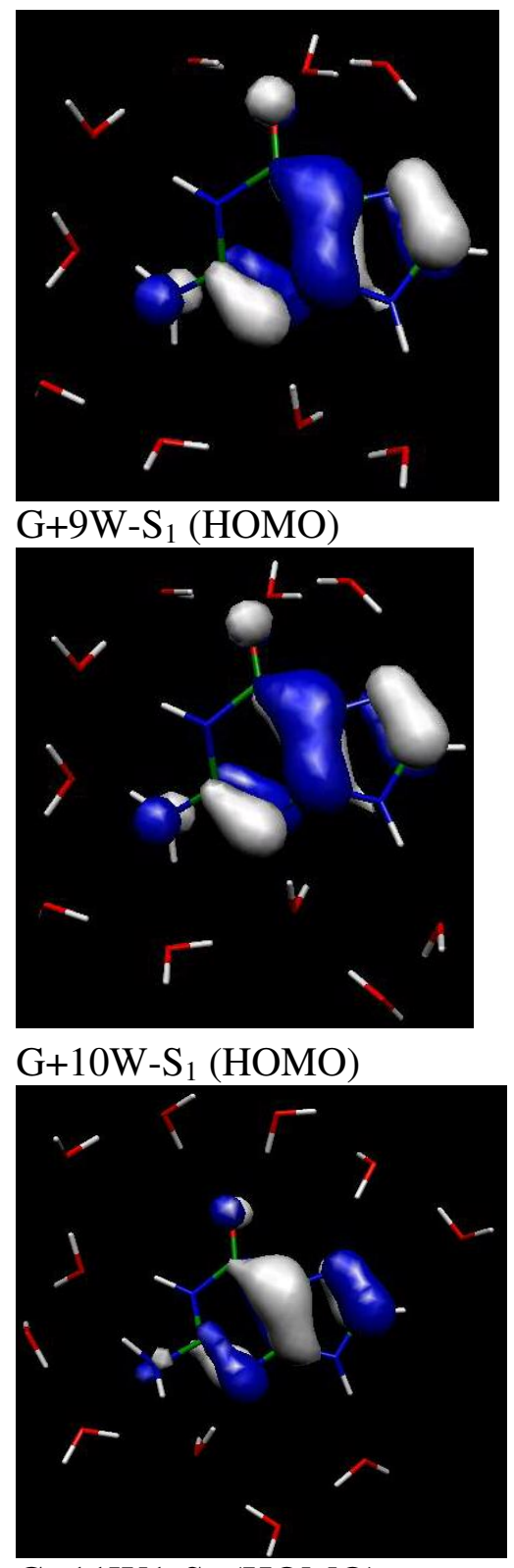

G+11W1-S 1 (HOMO)

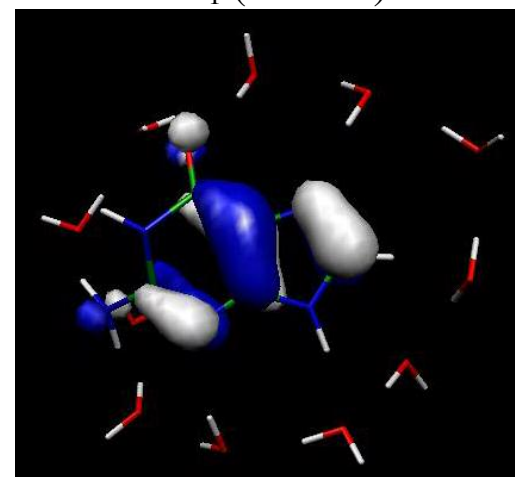

G+11W2-S 1 (HOMO)

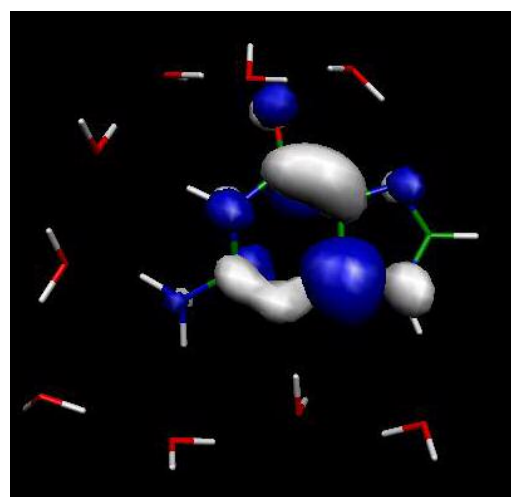

LUMO
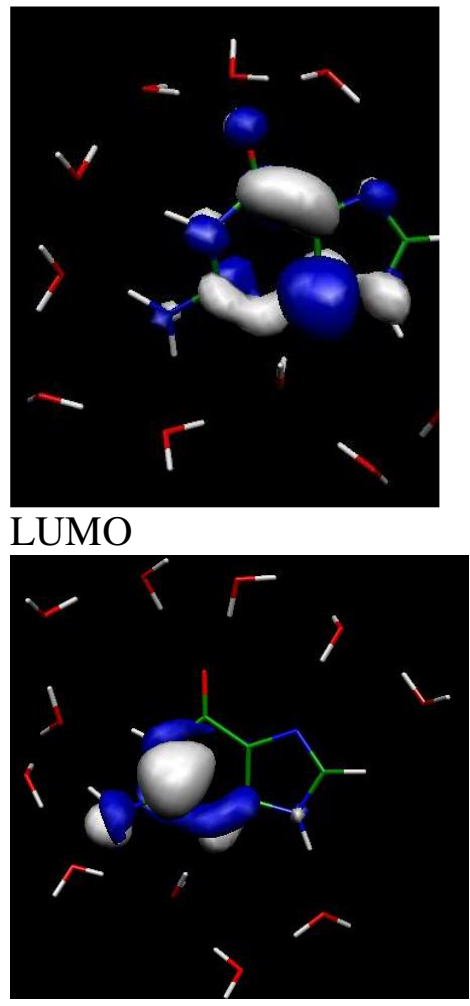

LUMO

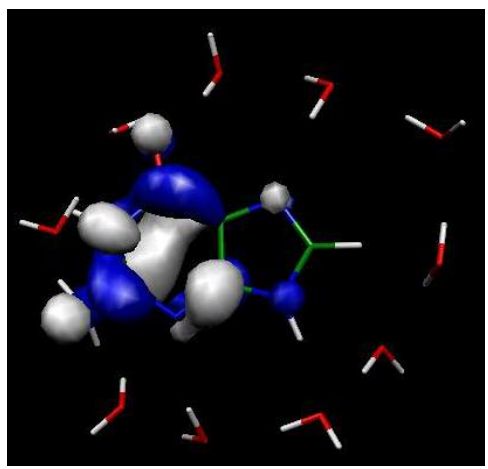

LUMO 

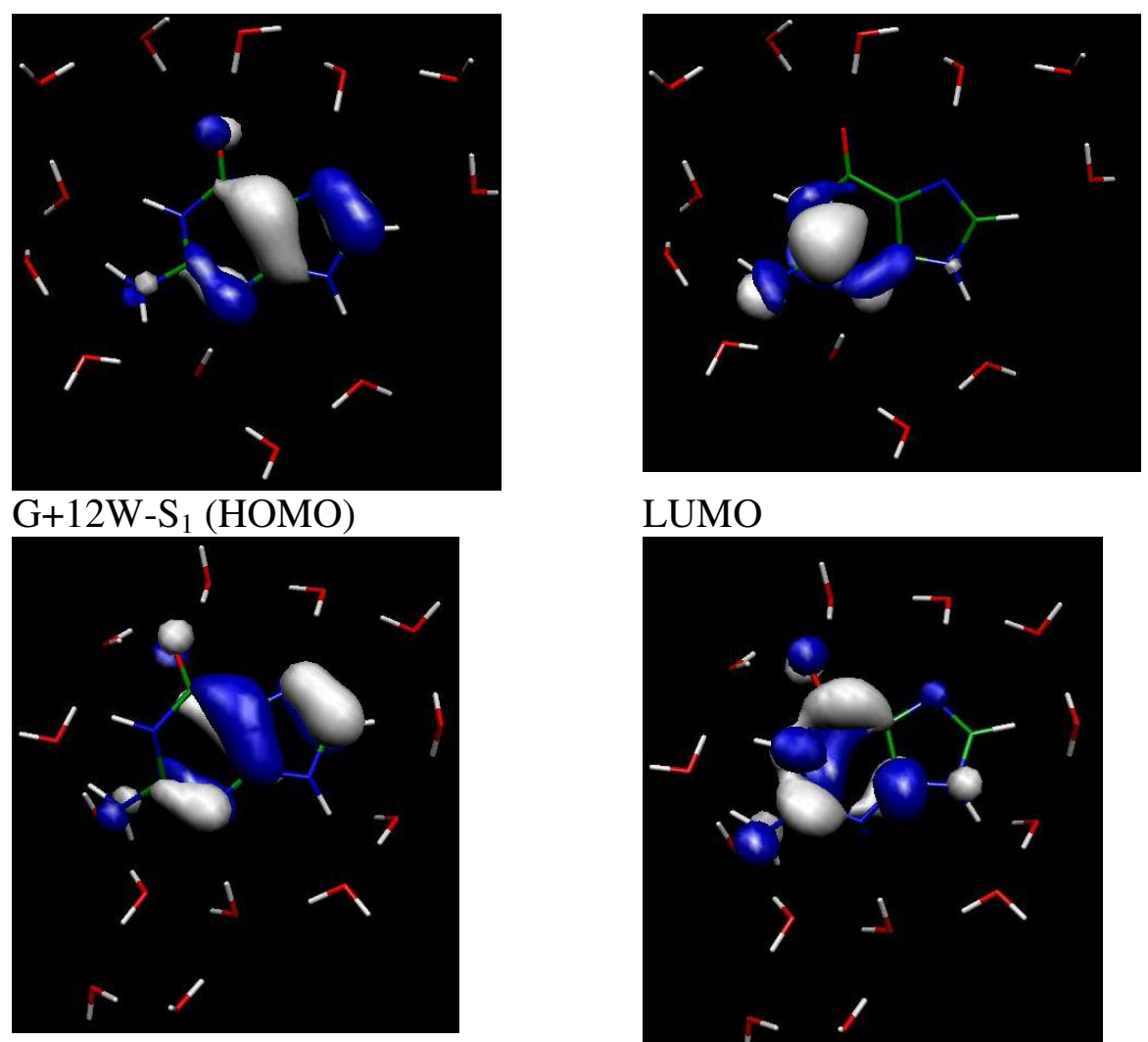

$\mathrm{G}+13 \mathrm{~W}-\mathrm{S}_{1}(\mathrm{HOMO})$

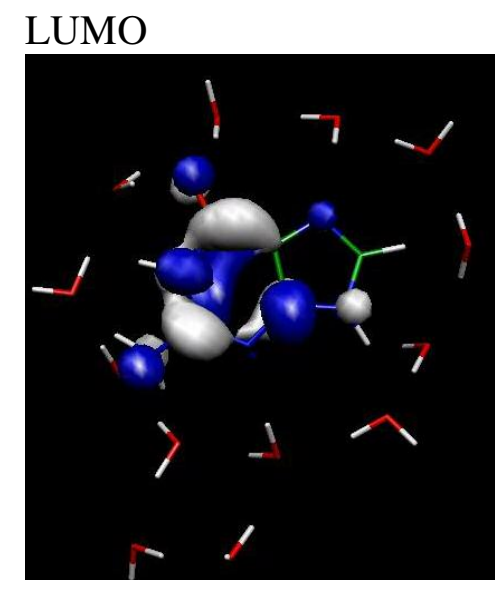

LUMO

Figure S1. HOMO and LUMO orbitals corresponding to the excited state optimized geometry of hydrated complexes of guanine. 